\title{
FLEXIBILIZACIÓN CUANTITATIVA (FC) Y SU APLICACIÓN EN ECUADOR
}

\section{QUANTITATIVE EASING (FC) AND ITS APPLICATION IN ECUADOR}

\begin{abstract}
Juan Francisco Sánchez Guerrero, Mgs. Magíster en Finanzas y Proyectos Corporativo. Docente Titular en la Facultad de

Ciencias Económicas Universidad de Guayaquil jfsg1178@hotmail.com

Bella del Rocío Garabiza Castro, Mgs. Magíster en Finanzas y Proyectos Corporativos. Docente Titular en la Facultad de Ciencias Económicas Universidad de Guayaquil bella.garabizac@ug.edu.ec
\end{abstract}

Alfonso Rafael Casanova Montero, PhD.

Doctor en Ciencias Económicas. Docente Titular auxiliar de la Facultad de Ciencias Económicas de la Universidad de Guayaquil. casanovamontero1954@gmail.com

\section{ARTÍCULO DE REVISIÓN}

Recibido: 12 de mayo de 2017

Aceptado: 13 de junio de 2017

\section{RESUMEN}

El objetivo general que rige el presente trabajo es informar sobre el concepto, desventajas, ventajas, y aplicaciones de la flexibilización cuantitativa. La metodología utilizada fue el deductivo porque busca en los argumentos las bases para conocer y entender las relaciones entre los agentes involucrados en una flexibilización cuantitativa, 
y se dirige hacia lo inductivo por medio de la forma en cómo se aplicó en Ecuador. Al final se concluye que, sin moneda nacional, con un sistema financiero de carácter público, y con un banco central sin autonomía, es factible aplicar la flexibilización cuantitativa por parte del gobierno, porque ayudó a canalizar los recursos, por un lado a canalizarlos al crédito, y por otro lado a cancelar pagos atrasados.

Palabras claves: flexibilización cuantitativa, ecuador, ventajas, desventajas.

\section{ABSTRACT}

The general objective that governs the present work is to inform about the concept, disadvantages, advantages, and applications of the quantitative easing. The methodology used was the deductive because it seeks in the arguments the bases to know and understand the relations between the agents involved in a quantitative easing, and it is directed towards the inductive through the way in how it was applied in Ecuador. In the end, it is concluded that, without national currency, with a public financial system, and with a central bank without autonomy, it is feasible to apply quantitative easing by the government, because it helped to channel resources, on the one hand to channel them to the Credit, and on the other hand to cancel late payments.

Keywords: quantitative easing, Ecuador, advantages, disadvantages.

\section{INTRODUCCIÓN}

La flexibilización cuantitativa no es estudiada desde la academia ecuatoriana, y tampoco es informado desde los medios locales de comunicación. Prácticamente, hay que recurrir a fuentes extranjeras para conocer, identificar, entender y relacionar sus ventajas y desventajas. Además, es necesario conocer el rol de los bancos centrales y el escenario donde actúan, porque el banco central ecuatoriano es relativamente diferente al resto.

La flexibilización cuantitativa tiene criterios detractores como también aquellos que la defienden. No obstante, las potenciales economías capitalistas del mundo como Japón, Reino Unido y Estados Unidos la han implementado varias veces. Siempre como una medida anticrisis, para reactivar el consumo y la inversión, reducir la deflación y aumentar la tasa de interés que se encuentran cercanas a cero. 


\section{REVISIÓN TEÓRICA}

\section{El Banco Central y su presencia en la economía.}

Un Banco Central (BC) es una institución que vela por el manejo económico en su país de residencia. Para Francisco Mochón el BC tiene algunos objetivos como:

1) Administrar y custodiar las reservas de oro y divisas, 2) Ser un banco del Estado, 3) Actuar por medio de la política monetaria, 4) Ser banco de bancos, 5) Emitir, suministrar, retirar y canjear dinero legal de la economía y, 6) Evitar la inflación y deflación. (Mochón, 1995, pág. 284)

Cada objetivo mencionado se compone de otros objetivos específicos. Los cuales forman un gran entramado de acciones y medidas propias de las políticas: monetaria, cambiaria, financiera y crediticia; y juntas, actúan como "brazos hermanos" de la política fiscal. El caso del Banco Central del Ecuador (BCE), a pesar de seguir como institución importante, ha sufrido algunas modificaciones en su gestión económica. A partir del año 2000, después de adoptar al dólar como moneda circulante, medio de pago y unidad de cuenta en el territorio nacional, representó el costo de sacrificar la moneda nacional, y arrastro a la extinción algunas labores básicas de la entidad. Por ejemplo, la emisión de moneda local y su control.

Así, la base monetaria (BM) (Dinero de alta potencia) de una economía determinada "consta de efectivo (monedas y billetes) y depósitos que tienen los bancos (privados) en los bancos centrales" (Dornbusch, Fischer, \& Startz, 2008, pág. 395); y cuyo control es responsabilidad inherente del BC. En el caso de la BM en Ecuador se compone por la oferta monetaria nacional (M1), y sumando el cuasidinero se obtiene la liquidez total (M2) (BCE, 2011, pág. 13). Sin embargo, el hecho de ya no poseer al sucre como moneda propia no quiere decir que no exista M1. Al contrario, sí existe oferta monetaria pero en divisa extranjera y fracciones ecuatorianas del mismo. En tiro gracioso, es una especie de "M1 mixta (importada y nacional)".

Siguiendo con Mochón, él también hace referencia a que los bancos centrales pueden tener una "acción pasiva o activa ante factores autónomos y controlables" (Mochón, 1995, pág. 289). En otras palabras, activa (autónoma) porque puede modificar la base monetaria en su totalidad cuando sea necesario y oportuno, por medio del crédito a los bancos y la compra o venta de títulos de deuda; y pasiva (controlable) ante los factores 
autónomos como son el sector público y sector externo. Pero el BCE tiene actualmente, una actividad más pasiva que activa en el contexto de la teoría macroeconómica. El artículo 303 de la Constitución del Ecuador (2008, pág. 168) establece que, "la entidad es considerada únicamente como instrumento de la Función Ejecutiva y se aplica el derecho público; la política es responsabilidad del presidente; y para regular la circulación monetaria será por medio de la ley”. En resumidas cuentas, el BCE no es autónomo ni independiente. Pero mantiene otras funciones de importancia, entre ellas el encaje bancario.

El encaje bancario consiste, según dicta la doctrina económica, el BC tiene en su poder una proporción determinada de los depósitos que tienen los bancos comerciales (Dornbusch, Fischer, \& Startz, 2008). Respecto a la proporción del encaje bancario en Ecuador, el artículo 2 de la resolución No. 302-201 6-F dice que "es del 5 \% para aquellos bancos que superen activos por un monto de 1000 millones de dólares; y el resto de bancos que no superen, solo el 2 \%” (Junta Monetaria y Financiera, 2016, pág. 2).

\section{La Flexibilización Cuantitativa (FC)}

La flexibilización cuantitativa es una medida aplicada en contexto de la política monetaria y es responsabilidad entre el gobierno y el banco central. En pocas palabras, la FC es la compra de activos financieros. En otras palabras "en vez de pedir crédito usualmente con la emisión de bonos, el gobierno, por medio del banco central, crea dinero....... y lo canaliza a bancos y a otras entidades financieras a las que se propone ayudar” (Keeley \& Love, 2012, págs. 97-98)

También Blanchard, Dell'Aricci y Mauro (2014) hacen mención al respecto de la FC que: puede pensarse como una combinación de flexibilización dirigida (compra de activos de largo plazo, financiados con la venta de activos de corto plazo) y la expansión monetaria convencional (la compra de activos a corto plazo con dinero del banco central). (pág. 11)

Para Mario Draghi, quien es el actual presidente del Banco Central Europeo dijo al respecto que, "es un programa de estímulo cuyo elemento principal es la compra de deuda pública a modo de inversión por parte de un banco central", (Cardo, 2014), debido 
a que, en el momento de comprar deuda inyecta dinero en los bancos privados, y esto se vuelve información atractiva que se refleja en los mercados financieros.

Así como es defendida, también es criticada. Pero en esencia pretende, estimular al consumo por parte de las familias e incentivar la inversión privada y pública. Pero su mecanismo de ejecución es diferente a la versión tradicional (operaciones de mercado abierto), al momento de influir en la base monetaria, la tasa de interés, la tasa de cambio, y la inflación. Debido, “(...) al momento en que un banco central compra activos financieros en el mercado, sea a particulares o del Estado, y éste realiza el desembolso de dinero, lo que provoca un aumento en la base monetaria" (Dornbusch, Fischer, \& Startz, 2008).

La diferencia entre una operación de mercado abierto y una $\mathrm{FC}$, es que en este último, un banco central no compra activos financieros al Estado ni a los particulares, si no a los bancos privados.

Porque el rol de los bancos centrales no es dar ni un solo centavo a los gobiernos, ya que pueden crear hiperinflación (...). En cambio, un aumento en los depósitos bancarios se compensa por medio del encaje bancario, y el restante puede ser redirigido al crédito, sean estos: productivo, consumo, hipotecas, etc. (Hudson, 2015)

Por ello, la flexibilización cuantitativa -conocida en su lenguaje anglosajón como Quantitative Easing- da mucho de qué hablar. Debido a la política implementada desde el FED en el año 2008 a raíz de la crisis financiera. Y dicha operación ha sido membretada como "no ortodoxa".

\section{Desventajas de la FC}

Es el razonamiento de Hudson que invita a reflexionar sobre la FC. El simple hecho de aumentar los depósitos en los bancos privados, es donde la FC ejecutada por el BC se la considera "poco o nada convencional". Porque existen riesgos en el largo plazo al generar fuerte inflación.

Por ejemplo, Álvarez se pronuncia desde el Instituto Español de Estudios Estratégicos (IEEE) donde menciona algunos efectos por medio de la FC: 
Induce a una devaluación del tipo de cambio, muestra señales de un posible aumento de la inflación, repercute en pérdida de valor real para la divisa, y sería una atractiva oportunidad para los especuladores financieros en el mercado de divisas ofertando derivados e instrumentos sofisticados. Además, puede haber posturas contrarias entre los países en desarrollo. (Álvarez, 2013).

Respecto a este punto en particular, se debe más a la discrepancia por el manejo de la economía. Aquella que parte de la visión clásica versus aquella que parte de la visión no clásica. El punto en cuestión es el aumento en el nivel de precios. Porque, la teoría cuantitativa del dinero dicta que un aumento en la base monetaria en una economía es fuente de inflación, porque "el nivel de precios varía proporcionalmente a la cantidad de dinero" (Argandoña, 1990). Y ésta se hace notar en los precios de los bienes y servicios.

Supongamos que todas las afirmaciones por parte de Álvarez no se efectivicen. Bajo dicha premisa, igual no se descartan los posibles riesgos que se encontrarían latentes a causa de la FC. De la misma manera, el autor menciona al respecto algunos escenarios:

1) La infravaloración de la inflación puede ser generada por el aumento de dinero; 2) Los bancos no otorgarían créditos hasta obtener los ingresos por concepto de la venta de activos, y si reciben los ingresos, los desviarían a financiación o especulación en materia primas en los países emergentes (capitales golondrinas); 3) Puede darse una desigual distribución de la riqueza, beneficiando a las rentas más altas; 4) La moneda fuerte puede peligrar en su carácter de reserva global y por tanto su utilización por parte de países en desarrollo se convertiría en divisa de reserva; 5) Se incubaría una crisis si se arrepienten y desean retirarse de la inversión de la FC; y por último 6) Habría una devaluación competitiva generalizada que, impulsada por el proteccionismo, crea incertidumbre sobre el tipo de cambio y tendería a destruir el comercio internacional (Álvarez, 2013)

Otro aspecto desfavorable a considerar también puede ser: "la utilización para calcular obligaciones futuras de los fondos de pensiones, los rendimientos bajan y las obligaciones se elevan, porque los fideicomisos de pensiones estiman su valor en función del precio de los bonos" (Keeley \& Love, 2012). 
Aunque a simple vista esto pareciese cierto, puede haber algo de exageración, pero no por eso se puede descartar. La cuestión es, si estas ventajas o riesgos pueden darse en una economía con tipo de cambio fijo.

\section{Ventajas de la FC}

En cambio, por el lado viable de la FC existen otros enfoques que intentan defender su aplicación. Por ejemplo, el Presidente actual del Banco Central de Europa, Mario Draghi, declaró en una entrevista lo siguiente:

Es una buena medida para que entre liquidez en los bancos privados y así parar la deflación, y para que los bancos vuelvan a dar crédito a las familias y empresas; ello es necesario para generar empleo, crecimiento económicos, y se equilibre la oferta y demanda... (Cardo, 2014)

Su funcionamiento es una cadena de acontecimientos que se espera que se desencadenen simultáneamente.

El banco central al comprar deuda publica en poder de los bancos privados aumente la base monetaria de la economía nacional. Para que esto no afecte en el nivel de precios, se corrige con el Coeficiente de Caja impuesto a las instituciones financieras. El dinero sobrante por la venta puede ir a financiar fondos de inversión foráneos, convirtiéndose en el corto plazo, un impulso o una restricción en actividad económica, con una intención implícita que es contrarrestar la deflación.

\section{Algunas experiencias con el FC.}

La presencia de la FC no es una medida monetaria nueva. Los países asiáticos fueron los primeros en romper con la tradición ortodoxa. Seguido están las potencias anglosajonas como Estados Unidos e Inglaterra. Todos con un mismo fin en común: reducir la deflación, estimular el consumo y la inversión.

El Banco Central de Japón desde el año 2001 comenzó a comprar activos financieros a los bancos privados para que amplíen y concedan líneas de créditos, con la intención de estimular el consumo y la inversión. Sin embargo, en economía, no todo sale como se planea. "Si bien la FC contribuyó a un ambiente agradable para las inversiones y liderazgo corporativo y empresarial, no contrarrestó del todo los impactos en la 
economía. La razón fue la debilidad del sistema bancario, arrastrada por la crisis financiera” (International Monetary Fund, 2011, pág. 27). Para el año 2013, duplicó la base monetaria por medio del recompra de deuda para aumentar moderadamente el nivel de precios y así contrarrestar la deflación. Y en el 2016, Haruhiko Kuroda gobernador del Banco de Japon (Boj) planteó comprar bonos de renta fija de manera “ilimitada" (El Español, 2016). "La meta del índice de precios al consumidor intenta alcanzar 2 puntos porcentuales por medio de una expansión de la base monetaria, con la condición que haya estabilidad; y de manera que contrarreste la especulación y aumente la tasa de interés negativa que se registró en $0.1 \%$, convirtiéndola a en una meta ambiciosa" ( El Economista, 2016).

En el caso de Estados Unidos, la FC fue adoptada en pleno rescate hacia los bancos privados a causa de la crisis financiera del año, periodo 2008-2012, y la cual tuvo débiles repercusiones al interior (Stiglitz, 2016). Y consistió en un programa de rescate en tres fases.

La primera inyección de efectivo fue en el año 2008; la segunda inyección fue en el 2010; y la tercera y última fue en el año 2011 por medio de la compra de los bonos tipo Mortange Backed Securities que son aquellos valores que se respaldan por medio de hipotecas (Hausken \& Ncube, 2013, pág. 1).

Siguiendo el caso del país norteamericano y volviendo con Stiglitz, él hizo algunas reflexiones sobre los resultados de esta medida económica:

En Estados Unidos entre 2009 - 2011 el dinero por la venta de activos se desvió de su meta que era el gasto de bienes y servicios, según lo que esperaba el FED. Los canales de crédito importantes estaban bloqueados; y el dinero salía hacia economías fuertes y con ambientes de inflación. El efecto fue menoscabado cuando alzó el precio del dólar, la tasa de interés hipotecario bajaron, los monopolios se hicieron más fuertes con los ganancias extras, y los ricos aumentaron su consumo en importaciones ostentosas. (Stiglitz, 2016).

Inglaterra fue otro ejemplo de la aplicación de la FC. Durante los años 2009 y 2011 (Álvarez, 2013)".

La aplicación de la agresiva expansión monetaria, Hausken et al. (2013) sumó 375 billones en libras esterlinas, siendo la compra de bonos estatales más 
grande efectuada en dicho país; además de "reequilibrar" los portafolios y garantizar el rendimiento de los activos llamados dorados (gilts); y sí contribuyó a la reducción de inflación. (págs. 1 - 3)

\section{Experiencia Ecuatoriana}

Las características que presenta la economía ecuatoriana hacen que la implementación de una FC sea interesante y especial. El país no tiene moneda propia desde el año 2000. Solo cuenta -como se mencionó al principio-- con fracciones de dólar acuñadas en territorio nacional. Cuenta con una nueva Carta Magna aprobada en referéndum popular, donde se contempla, no una economía de mercado pura ni una economía social de mercado, más bien es denominada Economía Social y Solidaria. El Estado es garantista de varios derechos, y el actor gubernamental está al frente de la mayoría de decisiones de políticas económicas por medio del ejecutivo.

El país también enfrentó una crisis y una recesión. La primera en el año 2008 originado en el mundo financiero mundial, y la segunda en el 2014 por la baja del precio en las materias primas (commodities). La baja en el precio del barril del petróleo en los mercados mundiales afectó considerablemente en el presupuesto nacional, y por ende en la asignación de recursos para cubrir gasto público. Además, por la falta de ingresos petroleros generó iliquidez en la economía.

En el contexto del comercio mundial, el gobierno tomó la decisión de contrarrestar las importaciones para proteger el empleo nacional, y resguardar la dolarización. Para ello implementó aranceles a ciertos bienes importados y un impuesto para la salida de divisas evitar la salida de dólares. Pero aun así, los mercados fronterizos, en especial en el norte del país, fronterizo con Colombia, ha salido fuertemente afectado, principalmente por la devaluación monetaria del peso colombiano, haciendo más baratos sus exportaciones y ganando competitividad.

Dentro de este marco económico referencial, en el país se implementó dos programas de FC. Según Weisbrot, Johnston, y Merling (2017), "hubo dos programas de FC. El primero fue en el periodo 2011 - 2013 cuya suma ascendió a 1,7 billones de dólares; y el segundo fue en el 2014 - 2016 llegó a 2.4 billones de dólares". 
Lo particular del primer programa es la circulación de los recursos para canalizarlos al sector financiero. Por ejemplo, Weisbrot et al., "los bancos estatales emiten bonos para que el Banco Central de Ecuador los compre por primera vez, y con aquel ingreso compran bonos del gobierno o son destinados hacia el sector privado por medio del crédito productivo". El segundo programa fue más una ayuda a las finanzas gubernamentales. Porque "el gobierno emitió bonos y fuero comprados directamente por el banco central, y con esto se pagó las cuentas atrasadas que se agravaron por la recesión mundial, y afectó a toda la cadena de proveedores”. (Weisbrot, Johnston, \& Merling, 2017)

Es así que, sin moneda propia y con dolarización, hay límites para aplicar la FC, pero no se puede negar que es un mecanismo importante de la política anticíclica.

\section{CONCLUSIONES}

En teoría, el banco central debe ser autónomo e independiente porque es la entidad responsable de la política crediticia, monetaria, financiera y cambiaria. Pero en la realidad ecuatoriana, al decidir eliminar la moneda local y adoptar una divisa extranjera como medio circulante en la economía doméstica desde el año 2000, la presencia de la entidad quedó reducida a ser un instrumento y formar parte del sector público vía constitución. Por lo que, ya no puede actuar de manera sustancial en M1, pero aún puede influir en $\mathrm{M} 2$.

La flexibilización cuantitativa no es una medida nueva y ha sido implementada por las mayores economías capitalistas del mundo. Aunque puede ser considerada nada tradicional u ortodoxa, porque rompe con la lógica tradicional que tienen los bancos centrales en sus operaciones de mercado abierto. Además, puede ser aplicable en economías que tengan moneda propia o moneda adoptada.

En nuestro país, la aplicación de la FC, pudo lograrse por medio de la banca pública. Por un lado, contribuyó a ampliar el abanico de opciones crediticias, y por otro lado formó parte de la competencia de la banca privada. Esto es importante recalcar, debido a que tradicionalmente se piensa que el sector financiero debe estar alejado de los fines del público y solo se maneja dentro de lo privado, concentrando los recursos en un solo 
lado y calificando a pocos como aptos para obtener crédito. Pues contrariamente, los gobiernos también pueden proveer las condiciones para generar el sector financiero público. Solo es necesario interés político.

\section{REFERENCIAS BIBLIOGRÁFICAS}

El Economista. (21 de septiembre de 2016). El Economista. Obtenido de Banco de Japón transforma su política monetaria: http://eleconomista.com.mx/mercadosestadisticas/2016/09/21/banco-japon-transforma-su-politica-monetaria

Álvarez, G. (24 de 9 de 2013). Instituto Español de Estudios Estratégicos. Obtenido de La generalización del Quantitative Easing y sus repercusiones en la estabilidad financiera global: http://www.ieee.es/Galerias/fichero/docs_opinion/2013/DIEEEO882013_QuantitativeEasing_G.AlvarezRubial.pdf

Argandoña, A. (1990). El pensamiento económico de Milton Friedman. Madrid: IESE Business School. Obtenido de http://www.iese.edu/research/pdfs/DI-0193.pdf

BCE, B. C. (04 de 2011). Banco Central del Ecuador. Obtenido de Metodología de la Información Estadística del Mensual: https://contenido.bce.fin.ec/documentos/PublicacionesNotas/Catalogo/IEMensual/meto dologia/METODOLOGIA3RAed.pdf

Blanchard, O., Giovanni, D., \& Mauro, P. (2014). Repensar la politica macroeconómica II: ir a los detalles. s/n: International Monetary Fund .

Cardo, R. (04 de 12 de 2014). La Opinión de Telerife. Obtenido de http://www.laopinion.es/finanzas-personales/2014/12/04/flexibilizacioncuantitativa/579126.html

Constituyente, A. (2008). Constitución de la República del Ecuador. Quito: Registro Oficial de la Asamblea.

Dornbusch, R., Fischer, S., \& Startz, R. (2008). Macroeconomía. Décima edición. México: McGraw-Hill Irwin. 
El Español. (11 de noviembre de 2016). Obtenido de La última locura de Haruhiko Kuroda (BoJ): intervención total del mercado de deuda.: http://www.elespanol.com/economia/20161117/171483067_0.html

Hausken, K., \& Ncube, M. (2013). Quantitative Easing and Its Impact in the US, Japan, the UK and Europe. United States: Springer Science \& Business Media.

Hudson, M. (3 de 3 de 2015). counterpunch. Tells the facts, names the names. Obtenido de Quantitative Easing for Whom?: http://www.counterpunch.org/2015/03/13/quantitative-easing-for-whom/

International Monetary Fund. (2011). Japan: Selected Issues. s/n: International Monetary Fund.

Junta Monetaria y Financiera. (24 de 11 de 2016). Junta Monetaria y Financiera. Obtenido de Resolución No. 302-201 6-F: http://www.juntamonetariafinanciera.gob.ec/PDF/Resolucion-302-2016-F.pdf?dl=0

Keeley, B., \& Love, P. (2012). Esenciales OCDE. De la crisis a la recuperación. Causas, desarrollo y consecuencias de la Gran Recesión. s/n: OECD Publishing.

Mochón, F. (1995). Principios de economía. Madrid: McGraw Hill/Interamericana de España, S. A. U.

Stiglitz, J. (2016). El Euro. Cómo la moneda común amenaza el futuro de Europa. Madrid: Penguin Random House Grupo Editorial España.

Weisbrot, M., Johnston, J., \& Merling, L. (febrero de 2017). Center for Economic and Policy Research. Obtenido de Decade of Reform: Ecuador's Macroeconomic Policies, Institutional Changes, and Results: http://cepr.net/publications/reports/decade-of-reform-ecuador-s-macroeconomicpolicies-institutional-changes-and-results 